\title{
Laser-induced forward transfer of focussed ion beam pre-machined donors
}

\author{
K.S.Kaur ${ }^{1, *}$, M.Feinaeugle ${ }^{1}$, D.P.Banks ${ }^{1}$, J.Y. Ou ${ }^{1}$, F. Di Pietrantonio ${ }^{2}$, E. Verona ${ }^{2}$, \\ C.L.Sones ${ }^{1}$, R.W.Eason ${ }^{1}$ \\ ${ }^{1}$ Optoelectronics Research Centre, University of Southampton, Southampton SO17 1BJ, UK. \\ ${ }^{2}$ Institute of Acoustics and Sensors- CNR 10000133 Rome - Italy
}

\begin{abstract}
In this paper we report femtosecond laser-induced forward transfer (LIFT) of pre-machined donor films. $1 \mu \mathrm{m}$ thick zinc oxide $(\mathrm{ZnO})$ films were first machined using the focussed ion beam (FIB) technique up to a depth of $0.8 \mu \mathrm{m}$. Debris-free micro-pellets of $\mathrm{ZnO}$ with extremely smooth edges and surface uniformity were subsequently printed from these premachined donors using LIFT. Printing results of non-machined $\mathrm{ZnO}$ donor films and films deposited on top of a polymer dynamic release layer (DRL) are also presented for comparison, indicating the superior quality of transfer achievable and utility of this premachining technique.
\end{abstract}

Keywords : laser induced forward transfer (LIFT), focussed ion beam (FIB), ZnO, DRL

*Corresponding author: Email: kak@orc.soton.ac.uk, Tel: +44 23805929091 


\section{Introduction}

Laser induced forward transfer (LIFT) is a versatile direct-write method for spatially selective printing of a wide range of materials [1-3]. In the conventional LIFT technique a thin film (usually of thickness $<1 \mu \mathrm{m}$ ) of the material (the donor) to be printed is deposited on top of a substrate (the carrier) which is transparent to the incident laser wavelength. A laser pulse is then focussed/imaged onto the carrier-donor interface which induces the necessary impulsive force to push the donor onto a substrate placed nearby (the receiver) either by melting it (for the case of a sufficiently thin donor ) [4,5] or ablating the top layer (the thick donor case) [6]. The complete melting or disintegration of the donor material during the transfer process is clearly a major drawback especially when printing is required of materials in solid and intact form (e.g. single crystals), oriented films, single domain or other pre-structured donors.

One technique that has met with considerable success to mitigate the above mentioned problems of damage to the donor layer is dynamic release layer (DRL)-LIFT. In DRL-LIFT a sacrificial layer called the dynamic release layer is introduced between the carrier and the donor film to avoid direct exposure of the donor to the incident laser. This DRL can be a metal $[7,8]$ or a polymer film [9, 10], a light-to-heat conversion layer as in laser induced thermal imaging (LITI) [11] or a specially engineered nanomaterial layer as in nanomaterial enabled laser transfer (NELT) [12]. These complementary LIFT methods have demonstrated the transfer of biomaterials $[8,10]$, conductive polymers [11], OLED pixels $[9,12]$ ceramics [13] and semiconductor bare dies [15]. Recently printing of $\mathrm{SiO}_{\mathrm{x}}$ was also reported by coating the receiver film directly onto the donor to reduce the debris associated with the deposits [15]. However these techniques do not eliminate one of the basic problems in LIFT printing, namely the inevitable shearing or ripping of the donor film at the boundary of the illuminated region, which may present an unacceptable limitation to the use of LIFT where edge quality is of prime importance, for example where multilayer donors are used. Spatially shaped multiple pulses to define weakened regions into the donor film before printing have been used to reduce this problem in another complementary LIFT technique called ballistic laser-assisted solid transfer (BLAST) [16]. Complete micro-dissection of biological samples prior to transfer to avoid contamination has also been reported in the literature [17].

In this paper we introduce a new variant to the LIFT technique that allows debris-free printing of solid donors in an intact form without using any DRL. The critical step is to premachine the donor using, in our case, focused ion beam (FIB) machining to encourage separation and transfer of predetermined shapes in intact and solid form. It is not necessary to 
machine throughout the entire depth of the donor however as this would prevent the laserinduced pressure build-up behind the material intended for transfer. For the optimum depth of pre-machining into the donor, uniform and clean deposits are printed onto the receiver. This technique should also significantly extend the thickness limitation for donor layers to beyond the current $\sim \mu \mathrm{m}$ level: with a maximum value of $\sim 1 \mu \mathrm{m}$ being reported to date using a triazene polymer (TP) DRL [18]. Zinc oxide ( $\mathrm{ZnO})$, an environmental-friendly lead free piezoelectric was chosen as the trial donor material for these LIFT experiments, and we report our first results below.

\section{Experiments and results}

The donor samples were prepared by sputtering $1 \mu \mathrm{m}$ thick films of $\mathrm{ZnO}$ on top of quartz substrates (the carrier) at a pressure of $3 \mathrm{mT}$ Torr and a temperature of $200^{\circ} \mathrm{C}$. FIB machining was used to etch circular features into $1 \mu \mathrm{m}$ thick sputtered donor films up to a depth of 0.8 $\mu \mathrm{m}$ with $5 \mu \mathrm{m}$ and $10 \mu \mathrm{m}$ diameters and centre-to-centre separation of $50 \mu \mathrm{m}$. We defined a parameter ' $\Delta$ ' to quantify the fractional etched depth for the donor:

$$
\Delta=\text { (milled depth) / (donor thickness) }
$$

The $\Delta$ value chosen for our trial demonstration of this FIB pre-patterning technique was 0.8 . It took $\sim 80 \mathrm{sec}$ to machine each ring at a current value of $2.8 \mathrm{nA}$ with a dosage of 2.81 $\mathrm{nC} / \mu \mathrm{m}^{2}$ per ring. These first samples were machined using $\mathrm{Ga}+$ ions only without the use of any gas for enhanced etching. If halogen containing gases were used, then the rate of etching can be increased considerably, reducing the pre-machining time to perhaps tens of seconds per ring. The value of 0.8 for $\Delta$ was chosen based on the requirement to mill away a substantial amount of material, without going to the extremes of $\Delta=1$. A previous trial had been carried out on other donors that included metals such as $\mathrm{Au}$ and $\mathrm{Cr}$, and $\Delta$ values exceeding 1.0 (i.e. FIB machining through the donor and into the carrier itself) had been tried on the basis that machining away the entire thickness would be the best strategy to ensure easy detachment [19]. Values for $\Delta$ that approach (or exceed) 1.0 however mean that any explosive propulsion force generated from LIFT is no longer constrained at the donor/carrier interface, and the propulsion force can leak around the milled slot. For this reason we chose a trial value of 0.8 , which was felt to represent a reasonable compromise between removing too much material, and not removing sufficient, so that debris would still present possible problems. 
In any future work, optimising the $\Delta$ value for a particular donor material would be an important step, as smaller values of $\Delta$ require less time for FIB machining. As it stands, the 80 seconds per feature is still a fairly long time. However, as will be seen later, this does result in side-walls for the LIFTed pellet that are extremely smooth, and for certain applications, where printing of a limited number of pellets is required, in a debris-free manner, this time may not be considered particularly restrictive. Figure 1(a) shows the SEM image of an array of machined ring patterns while fig. 1(b) shows a magnified image of one of the $5 \mu \mathrm{m}$ rings.
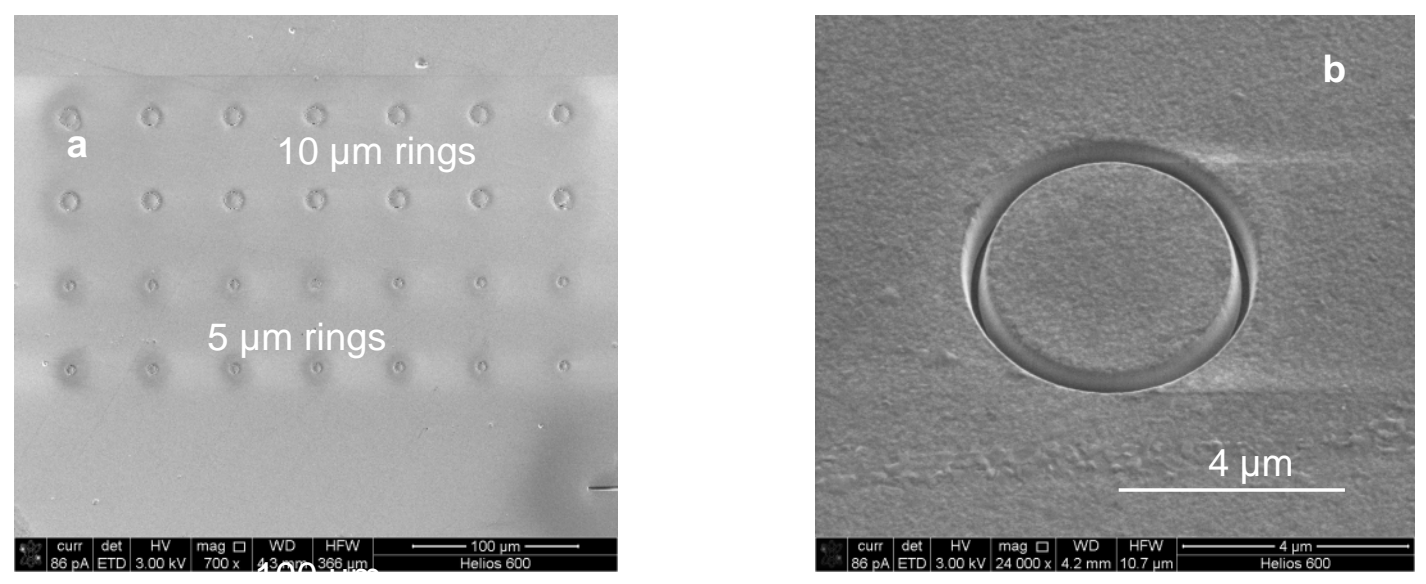

[Figure 1(a,b)]: (a) SEM images of the FIBbed ring patterns of $10 \mu \mathrm{m}$ (top two rows) and 5 $\mu \mathrm{m}$ (bottom two rows) diameters in $1 \mu \mathrm{m}$ thick $\mathrm{ZnO}$ donor film. (b) Shows the magnified SEM image of one of the machined $5 \mu \mathrm{m}$ rings.

For comparison $1 \mu \mathrm{m}$ thick $\mathrm{ZnO}$ films on top of TP as a DRL and $1 \mu \mathrm{m}$ thick $\mathrm{ZnO}$ samples without any prior machining were also prepared for LIFTing. For all the experiments a commercial mode-locked Ti: sapphire laser femtosecond laser was used. Single laser pulses with a Gaussian spatial profile $(800 \mathrm{~nm}, 150 \mathrm{fs}, \mathrm{FWHM} \sim 4 \mathrm{~mm})$ were first centred on a 450 $\mu \mathrm{m}$ diameter circular aperture resulting in a reasonably spatially uniform incident pulse profile with spot size of $\sim 12 \mu \mathrm{m}$. The beam size was chosen to be bigger than the FIBbed feature sizes $(5 \mu \mathrm{m}$ and $10 \mu \mathrm{m})$ for ease of alignment while printing. A highly de-magnified image of the aperture was then relayed onto the carrier-donor interface using a commercial micromachining workstation (New Wave UP266, USA) thereby printing micro-pellets of $\mathrm{ZnO}$ onto silicon $(\mathrm{Si})$ receivers at a donor-receiver separation of $1 \mu \mathrm{m}$, using Mylar spacers. All the experiments were performed under a background pressure of $10^{-1} \mathrm{mbar}$. 

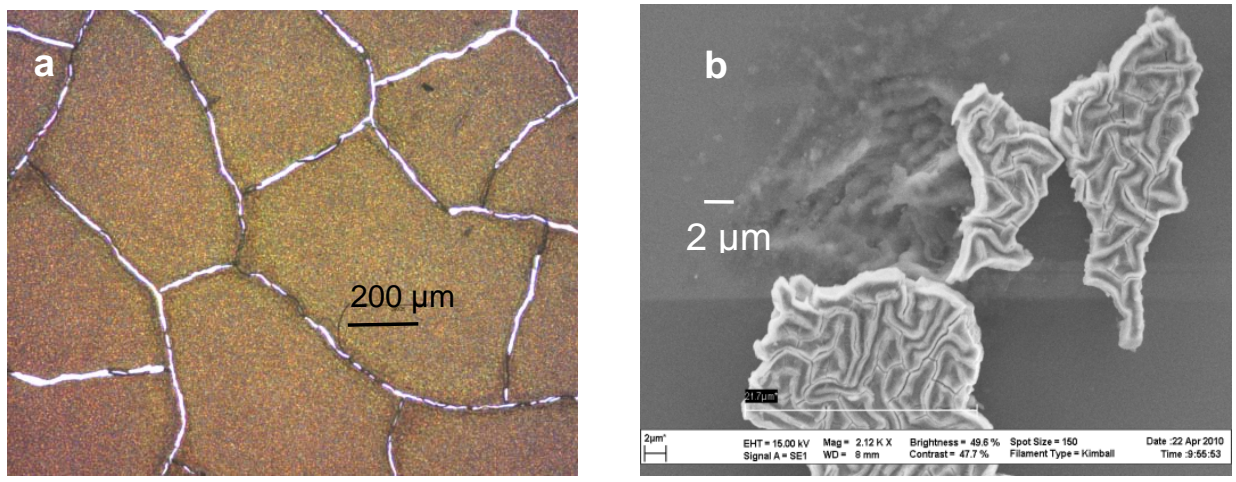

[Figure 2(a, b)]: Shows SEM image of $1 \mu \mathrm{m}$ thick $\mathrm{ZnO}$ donor film deposited on top of 360 $\mathrm{nm}$ thick TP (a). Shows SEM image of deposits printed from this donor film (b).

Figure 2 (a) shows an SEM image of a $1 \mu \mathrm{m}$ thick $\mathrm{ZnO}$ donor film sputtered on top of 360 nm thick TP. About $80-85 \%$ of the surface area of the donor films was cracked due to the thermal decomposition of TP while depositing the upper ZnO layer. Figure 2 (b) shows an SEM image of $\mathrm{ZnO}$ deposits printed using these films. Cracks were also clearly visible in the LIFTed deposits, and as the film was weaker around the cracks LIFTing preferentially occurred by rupture along the cracks leading to non-circular deposits. The bad quality of the donor films in this case resulted in shattered and irregular shaped pellets. We have also tried to deposit $\mathrm{ZnO}$ on TP-coated unheated carriers using other techniques including pulsed laser deposition (PLD). Again however, the quality of the resultant donor was less good than that achieved via sputtering on uncoated carriers. The degradation temperature of TP is of the order of $250^{\circ} \mathrm{C}$, and for thick films ( $\sim \mu \mathrm{m}$ thicknesses) prolonged exposure in a sputtering chamber can severely degrade the surface integrity. This is another reason why FIB premachining can be advantageous as it does not require any DRL use. It should in principle be applicable to print any donor material as the range of donors that can be printed using this technique is not limited by any temperature or chemical sensitivity issues as is the case for DRL-assisted LIFT. 

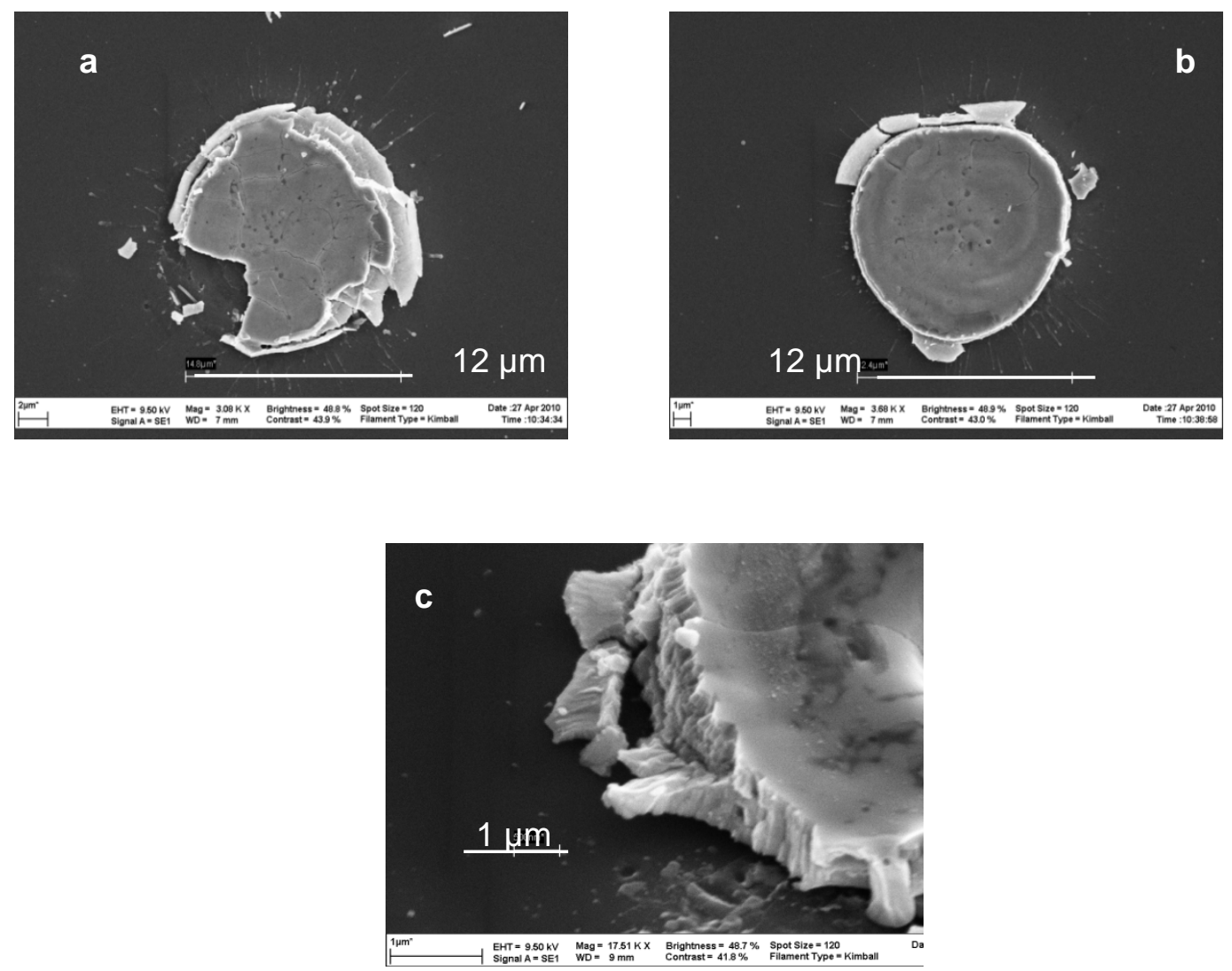

[Figure 3(a, b, c)]: (a) and (b) show the SEM images of $12 \mu \mathrm{m} \mathrm{ZnO}$ pellets printed form a non-machined $1 \mu \mathrm{m}$ thick donor. The shattering of the pellets during transfer produced non-uniform, irregular shaped and splashy deposits, clearly depicted in the images. (c) Shows the rough edges of the printed deposits.

Figure 3 (a) and 3 (b) show SEM images of typical and the best LIFTed deposits respectively of $12 \mu \mathrm{m}$ diameter $\mathrm{ZnO}$ pellets printed from a $1 \mu \mathrm{m}$ thick donor without any pre-machining, while fig. 3 (c) shows the edge quality of a typical deposit. The deposits were in general splashy, with considerable amounts of debris and with very rough and ill-defined edges and irregular shapes. However, the deposits from a pre-machined donor exhibited extremely good quality both in terms of surface uniformity and edge smoothness. Under the optimum conditions for LIFTing, there was no trace of any residual debris surrounding the transferred pellets. Figure 4 (a) and (b) show the SEM images of $10 \mu \mathrm{m}$ and $5 \mu \mathrm{m} \mathrm{ZnO}$ pellets respectively printed from a pre-machined donor. Note here that fig. 4(a) shows the result for LIFTing onto a $\mathrm{Si}$ substrate, whereas fig. 4(b) used a flexible compliant substrate (polystyrene). The adhesion of $\mathrm{ZnO}$ to the plastic receiver was not as good as onto Si. So far we have not made any adhesion tests for the printed pellets using this pre-patterning approach, and the use of flexible receivers appears to be non-optimum. Fortuitously however, 
the pellet LIFTed onto polystyrene has flipped over while dismantling the donor-receiver assembly, allowing SEM examination of the quality of the front surface, and edge quality around the machined rim.
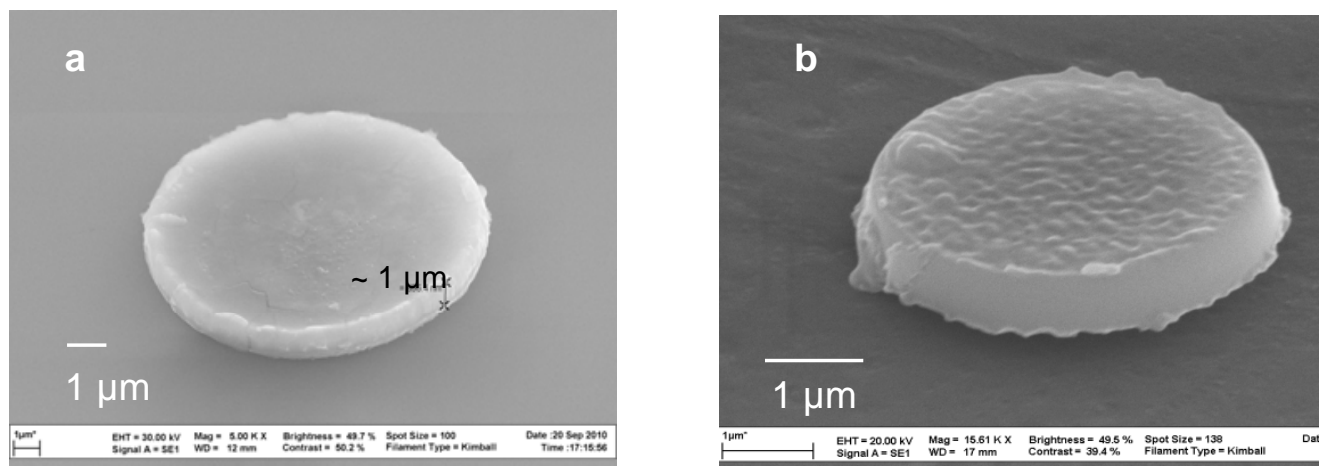

[Figure 4(a, b)]: $\quad$ SEM micrographs of $10 \mu \mathrm{m}$ (a) and $5 \mu \mathrm{m}$ (b) $\mathrm{ZnO}$ pellets printed onto Si and plastic receiver respectively from a pre-machined $1 \mu \mathrm{m}$ thick donor film.

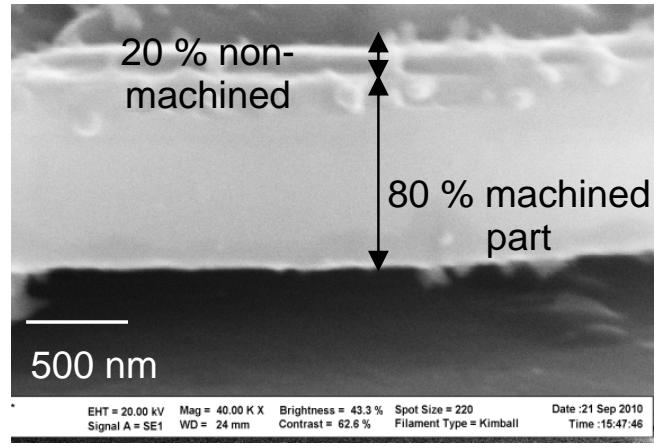

[Figure 5]: $\quad$ Magnified SEM image of the extraordinarily smooth edge of one of the deposits printed from the pre-machined $\mathrm{ZnO}$ donor.

Figure 5 shows an SEM image of the edge quality of a printed $10 \mu \mathrm{m} \mathrm{ZnO}$ pellet, and this degree of smoothness was routinely seen in all SEM pictures taken. The high edge quality pellets from the pre-machined $\mathrm{ZnO}$ donors were transferred at an incident laser fluence of $450 \mathrm{~mJ} / \mathrm{cm}^{2}$ (fig. 5) which is less than half the value $\left(\sim 1 \mathrm{~J} / \mathrm{cm}^{2}\right)$ used to transfer the shattered and rough edge quality deposits from the non-machined donors (fig. 3(c)). 
The difference in the quality of deposits transferred from donors with and without premachining prior to LIFT is clearly seen. For both cases the incident laser pulse provides the the required force to overcome the donor-carrier adhesion, but in the case of no prior machining, the donor pellet still has to shear itself from a considerable portion of the surrounding solid donor film, resulting in shattering during transfer, rough edges, irregular shapes and considerable and unavoidable debris. In case of the pre-machined donor, the area to be printed is already defined by FIB patterning which makes the transfer process much gentler. For cases where $\Delta$ approaches 1 , detachment can occur with minimal (in optimum cases zero) residual debris, and smooth, regular printing can occur.

It should be mentioned that while a $\Delta$ value of 0.8 has clearly produced impressive results, without further experimentation or modelling, it is not yet apparent which value produces the optimum level of printing fidelity. It is also likely that this parameter is both donor material and thickness specific. However, the striking difference in the quality of the deposits obtained clearly shows the great potential of this technique for printing thick and fragile donors in solid and intact format. Finally, we also mention that LIFTing of single crystal materials, such as those grown via PLD which usually requires substrate heating to temperatures in excess of $\sim 600^{\circ} \mathrm{C}$ to ensure single crystal thin film growth, and hence would be entirely unsuited to TP-DRL techniques, would also be an attractive prospect. FIB pre-machining is an ideal candidate to try here, and experiments are currently under way. We are not aware of any other reports of LIFTing of single crystal PLD-grown thin films, and this may well be a good example where FIB pre-patterning presents a useful, if slightly time-consuming process.

\section{Conclusions}

Micro-pellets of $\mathrm{ZnO}$ of excellent quality with extremely smooth and uniform edges were printed from pre-machined donors. The donor films were machined to a depth of $0.8 \mu \mathrm{m}$ by the FIB technique prior to LIFT. To the best of our knowledge, this is the first time that materials have been printed using the FIB pre-patterning LIFT technique. The initial results presented here were to validate the proof-of-principle for the technique, and further optimisation studies using donors with different $\Delta$ values, different thicknesses and donor materials are currently in progress. 


\section{Acknowledgement}

Financial support from the Engineering and Physical Sciences Research Council (EPSRC), UK, (under Grants no. EP/C515668/1 and EP/G060363/1) and the European community (under Grant no. 508581101, e-LIFT) are gratefully acknowledged.

\section{References}

[1] J. Bohandy, B.F. Kim, F.J. Adrian, Metal deposition from a supported metal film using an excimer laser, J. Appl. Phys. 60 (1986) 1538.

[2] K.D. Kyrkis, A.A. Andreadaki, D.G. Papazoglou, I. Zergioti, Recent Advances in Laser Processing of Materials, J. Perrière, E. Millon, E. Fogarassy, (Eds.), Elsevier, Amsterdam, 2006.

[3] A. Pique, D. Chrisey, R. Auyeung, J. Fitz-Gerald, H.Wu, R. McGill, S. Lakeou, P.Wu, V. Nguyen, and M. Duignan, A novel laser transfer process for direct writing of electronic and sensor materials, Appl. Phys. A. 69 [Suppl.] (1999) S279 - S284.

[4] D.P. Banks, C. Grivas, J.D. Mills, R.W. Eason, I. Zergioti, Nanodroplets deposited in microarrays by femtosecond ti:sapphire laser induced forward transfer, Appl. Phys. Lett. 89 (2006) 193107.

[5] D. A. Willis and V. Grosu, Microdroplet deposition by laser-induced forward transfer, Appl. Phys. Lett. 5713(2005) 90 - 96.

[6] I. Zergioti, A. Karaiskou, D. Papazoglou, C. Fotakis, M. Kapsetaki, D. Kafetzopoulos, Time resolved schlieren study of sub-pecosecond and nanosecond laser transfer of biomaterials, Appl. Surf. Sci. 247 (2005) 584- 589.

[7] B. Hopp, T. Smausz, Z. Antal, N. Kresz, Z.Bor, D. Chrisey, Absorbing film assisted laser induced forward transfer of fungi (trichoderma conidia), J. Appl. Phys. 96(6) (2004) 34783481 .

[8] P.Serra, M.Colina, J. M. Fernández-Pradas, Preparation of functional DNA microarrays through laser-induced forward transfer, Appl. Phys. Lett. 85 (9) (2004) 1639-1641.

[9] R. Fardel, M. Nagel, F. Nüesch, T. Lippert, A.Wokaun, Fabrication of organic lightemitting diode pixels by laser-assisted forward transfer, Appl. Phys. Lett. 91 (2007) 061103. [10] N. T. Kattamis, P. E. Purnick, R. Weiss, C.B. Arnold, Thick film laser induced forward transfer for deposition of thermally and mechanically sensitive materials, Appl. Phys. Lett. 91 (2007) 171120. 
[11] G. B. Blanchet, Yueh-Lin Loo, J.A.Rogers, F. Gao, C. R. Fincher, Large area, high resolution, dry printing of conducting polymers for organic electronics, Appl. Phys. Lett. 82 (3) (2003) 463-465.

[12] S.H.Ko,.Pan, S.G.Ryu, N.Misra, C.P.Grigoropoulos, H.K.Park, Nanomaterial enabled laser transfer for organic light emitting material direct writing, Appl. Phys. Lett. 93 (2008) 151110.

[13] D.P.Banks, K.Kaur, R.Gazia, R.Fardel, M.Nagel, T.Lippert, R.W.Eason, Triazene photopolymer dynamic release layer-assisted femtosecond laser-induced forward transfer with an active carrier substrate, Europhys. Lett. 83 (2008) 38003.

[14] S.A. Mathews, N.A. Chaipar, K. Metkus, A. Piqué, Manufacturing Microelectronics Using 'Lase-and-Place, Photonics Spectra 41(10) (2007) 70-74.

[15] J.Ihlemann and R.W.Schriever, Laser-induced congruent forward transfer of SiOxlayers, Appl. Phys. A. 101 (2010) 483-486.

[16] D.P.Banks, C.Grivas, I.Zergioti, R.W.Eason, Ballistic laser-assisted solid transfer (BLAST) from a thin film precursor, Opt. Exp. 16 (2008) 3249-3254.

[17] K.Schütze and G.Lahr, Identification of expressed genes by laser-mediated manipulation of single cells, Nature Biotechnology 16 (8) (1998) 737-742.

[18] K.Kaur, R.Fardel, T.C.May-Smith, M.Nagel, D.P.Banks, C.Grivas, T.Lippert, R.W.Eason, Shadowgraphic studies of triazene assisted laser-induced forward transfer of ceramic thin films, J. Appl. Phys. 105 (2009) 113119.

[19] D.P. Banks, Femtosecond laser induced forward transfer techniques for the deposition of nanoscale, intact, and solid-phase material, University of Southampton, personal communication, 2008. 